\title{
Flexible ureteroscopic holmium laser lithotripsy with PolyScope for senile patients with renal calculi
}

\author{
KE XU* ${ }^{*}$, JIE DING $^{*}$, BOWEN SHI ${ }^{*}$, YANYUAN WU and YUNTENG HUANG \\ Department of Urology, School of Medicine, Xin Hua Hospital Affiliated to \\ Shanghai Jiao Tong University, Shanghai 200092, P.R. China
}

Received October 8, 2017; Accepted June 11, 2018

DOI: $10.3892 /$ etm.2018.6369

\begin{abstract}
The present study reported the clinical experience of using a PolyScope with holmium laser lithotripter in managing renal calculi in senile patients. Between December 2013 and December 2016, 157 senile patients $(69.1 \pm 6.1$ years old) were treated with PolyScope holmium laser lithotripsy for renal calculi at Xin Hua Hospital (Shanghai, China). The mean stone digitized surface area was $154.2 \pm 57.7 \mathrm{~mm}^{2}$ (range, 55.8-478.3 $\mathrm{mm}^{2}$ ). A thorough medical history investigation, and laboratory and radiological examination were followed by proper preoperative management. The PolyScope was then passed into the renal cavities following the position of the ureteral access sheath, with laser energy maintained at 1.0-2.0 J, frequency $10-15 \mathrm{~Hz}$. The demographical and operative information of senile patients were compared with that of the 332 non-senile patients operated at the same period time. The average operative time was $54.6 \pm 13.2 \mathrm{~min}$ (range, 37-124 min). No major complications, including ureteral perforation or sepsis were encountered. The single session stone-free rate (SFR) was $81.5 \%$ and the overall SFR was $89.2 \%$, similar to that of the non-senile group. PolyScope combined with holmium laser lithotripter is safe and effective in managing renal calculi in senile patients compared with non-senile patients, with considerately high SFRs and low complication rates.
\end{abstract}

\section{Introduction}

The incidence of kidney stones has been increasing over the years (1). What's more, the incidence rate of kidney stone disease increases with aging $(2,3)$. Senile urolithiasis belongs to complex renal calculi disease, since the patients usually have

Correspondence to: Dr Yunteng Huang, Department of Urology, School of Medicine, Xin Hua Hospital Affiliated to Shanghai Jiao Tong University, 1665 Kongjiang Road, Shanghai 200092, P.R. China E-mail: huangyunteng@xinhuamed.com.cn

*Contributed equally

Key words: senile patients, renal calculi, flexible ureteroscope, PolyScope many comorbidities, such as chronic heart, lung, liver, kidney disfuction or severe urinary tract infection (4), adding extra difficulty to clinical treatment. Patients with kidney stones usually presented with hydronephrosis, back pain and fever and other symptoms, and need active and effective surgical treatment (5). Safe, timely and effective treatment of kidney stones can reduce serious complications such as kidney failure in senile patients, and significantly improve quality of life. This has brought urologists new requirements and challenges.

Nowadays, operation methods to treat kidney stones include open surgery, extracorporeal shock wave lithotripsy (ESWL), percutaneous nephroscope lithotripsy (PCNL) and ureteroscopic lithotripsy (URL). Traditional open surgery has lots of complications with considerate influence to renal function. The elderly with low body reserve capacity, reduced tolerance to surgery, slow postoperative recovery may develop many complications as the result of prolonged bed rest after open surgery $(6,7)$. In the past, limited by the hardware, kidney stones were treated by ESWL or PCNL with limited safety and efficacy (8). In recent years, with the development of flexible ureteroscope technical and auxiliary equipment (9), the flexible ureteroscopy (fURS) combined with holium laser lithotripsy is becoming mature and has gradually become an ideal choice for kidney stone. Compared with the ESWL or PCNL, especially in the treatment of senile kidney stone, its advantages are higher stone-free rate (SFR) and safer operation and fewer intraoperative and postoperative complications $(10,11)$. PolyScope is a novel removable modular flexible ureteroscope manufactured by Polydiagnost GmbH (Pfaffenhofen, Germany) (Fig. 1). The core components are the independent, optical, imaging modules, convenient to assemble or disassemble. The optical system has a soft yet tough synthetic metal wire net wrapped around for protection. The outer casing of the flexible ureteroscope is replaceable at a relatively low price. This eradicates the risk of cross-infection between operations, and enables the ureteroscope be used in successive procedures. The main advantage of PolyScope is its convenience for the replacement of consumable components to reduce the maintenance costs (12). We had already evaluated PolyScope against its conventional counterpart earlier (13). Yet its safety and effectiveness in treating senile patients, a special group of patients with urolithiasis, has not been reported so far. In the present study, from December 2013 to December 2016, 157 cases of senile patients with kidney stones were treated with PolyScope in Xin Hua Hospital Affiliated to Shanghai Jiao Tong 
University, School of Medicine (Shanghai, China). The results were satisfactory and are reported in the following sections.

\section{Patients and methods}

Patient characteristics. This is a retrospective study. A total of 157 patients (88 males and 69 females, aged from 60 to 82 years, mean age $69.1 \pm 6.1$ years old) with kidney stone who were admitted to Xin Hua Hospital Affiliated to Shanghai Jiao Tong University, School of Medicine from December 2013 to December 2016 were recruited for this study. The body mass index (BMI) ranged from 16.3 to 32.5 and the mean BMI was $23.4 \pm 2.9$. Nineteen patients had I degree of obesity $(25 \leq \mathrm{BMI}<30)$ and 5 patients had with II degree of obesity (BMI $\geq 30$ ). A total of 86 patients presented with hypertension, 26 with cardiovascular disease, 21 with anticoagulants or antiplatelet medications, 15 with diabetes, 38 with hydronephrosis, 13 with creatinine abnormality, 27 with COPD, 2 with horseshoe kidney, 2 with congenital solitary kidney. Thirty-two of the patients had been hospitalized and treated with ESWL, 17 with PCNL, 12 with fURS. Nineteen patients had previously had an indwelling double-J stent placed with ureteroscopic guidance in other hospital. All patients had been diagnosed by plain film of kidney-ureter-bladder (KUB) intravenous urography (IVU) and computed tomography (CT). In addition, 149 patients exhibited one-sided calculi, whereas 8 patients exhibited two-sided calculi. The total number of renal calculi was 245 and mean number of each side was $1.5 \pm 1.0$, including 61 renal pelvic calculi, 43 upper calyceal calculi, 55 middle calyceal calculi, 86 lower calyceal calculi. Digital surface area of each patient ranged from 55.8 to $478.3 \mathrm{~mm}^{2}$ (mean, $154.6 \pm 57.7 \mathrm{~mm}^{2}$ ). All patients' middle segment urine samples were collected for the germiculture and sensitivity test. The result was that 148 cases presented with no bacterial growth, 7 with Escherichia coli, 2 with Proteus. The information of 332 adult patients (185 males and 147 females, aged from 18 to 59 years, mean age $41.5 \pm 9.9$ years old) of the same period, who were also treated with PolyScope, were also analyzed and compared with that of the senile patients (Table I). The two groups had difference in comorbidity rate, including hypertension $(\mathrm{P}<0.01)$, cardiovascular disease $(\mathrm{P}<0.05)$, anti-coagulation $(\mathrm{P}<0.05)$ and $\mathrm{COPD}(\mathrm{P}<0.01)$. Other criteria were statistically similar between two groups.

Operational parameters. All patients were placed under spinal or general anesthesia (153 spinal anesthesia, 4 general anesthesia) in a lithotomy position with head tilted down. After location of the ureter, a zebra guide wire was inserted and a Wolf F8/9.8 rigid ureteroscope (Richard Wolf GmbH, Knittlingen, Germany) was placed into the ureter to reach the level of the pelvis. Following the removal of the rigid ureteroscope, a Cook F12/14 ureteral access sheath (UAS; Cook Medical, Inc., Bloomington, IN, USA) was positioned along the guide wire, as close to the level of the uretero-pelvic junction as possible, then PolyScope was inserted into the pelvis through the UAS. The power of the $220 \mathrm{~mm}$ holmium laser lithotripsy was set at 1.0-2.0 J. The 'nibble' approach was used to break and crumble the stones into fine granules measuring 2-3 $\mathrm{mm}$. Some stones with the diameter of 4-5 $\mathrm{mm}$ were removed with a stone basket, while some lower calyceal stones were translocated with the basket from the lower to the upper or middle calyx for improving

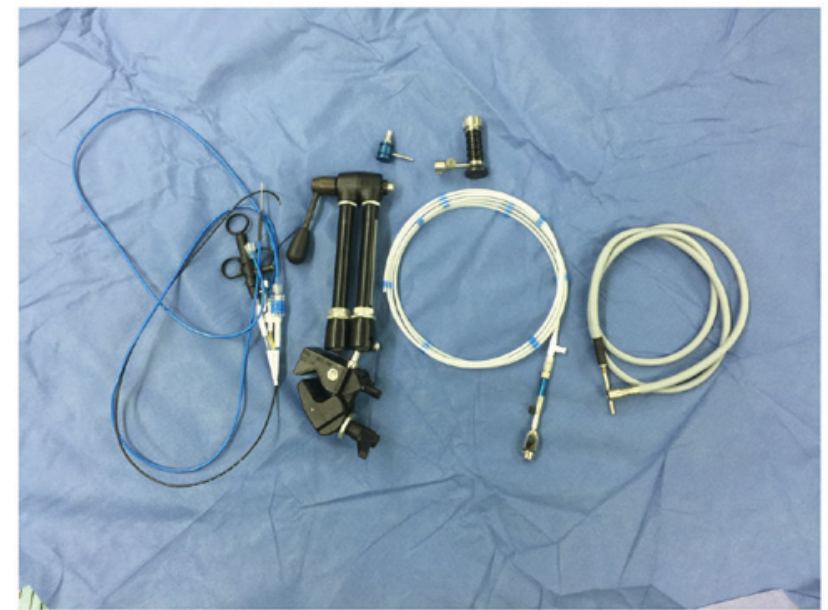

Figure 1. Components of PolyScope include a steerable endoscopic catheter system, image fiber, ocular, camera, light cables and a four-joint arm.

the efficiency of lithotripsy and also for protection of the optical fiber.

A F6 double-J stent was typically indwelled for 4 weeks following the surgery and a Foley urethral catheter was indwelled for 1-2 days. Plain film of KUB was performed 1 day subsequent to the surgery in order to determine the result of the lithotripsy and the position of the double-J stent. Postoperative follow-up to the hospital in a month was required. KUB or CT was performed and double-J stent removed.

Ethics approval and consent to participate. The Ethics Committee of Xin Hua Hospital Affiliated to Shanghai Jiao Tong University School of Medicine has approved the study and permitted the waiver of written informed consent from patients. The study protocol is also in accordance with the Declaration of Helsinki.

Statistical analysis. The data were analyzed using the SPSS 17.0 (SPSS, Inc., Chicago, IL, USA). Continuous data were presented as mean \pm standard deviation, and compared using Student's t-test. For categorical data, the values outside the brackets are the absolute patient number and the values inside the brackets are the percentage, and were compared using Chi-square or Fisher's exact test, and $\mathrm{P}<0.05$ was considered statistically significant. All tests were performed as a 2-tailed test.

\section{Results}

Lithotripsy was performed successfully in all 157 patients. 139 patients underwent one-stage lithotripsy and 18 patients two-stage. The duration of the surgery ranged from 37 to $124 \mathrm{~min}$ (mean duration, 54.6 $\pm 13.2 \mathrm{~min}$ ). Little bleeding was recorded and no ureteral perforation or damage was encountered. A total of 24 patients presented with fever following the surgery, their body temperature all returned to normal following anti-inflammatory treatment with either empirical antibiotics or according to the susceptibility results of preoperative urine culture. Three patients' urine color remained pink in one week after surgery, yet all became normal following the use of hemostatic drugs. The SFR was $81.5 \%$ for one-stage lithotripsy and $89.2 \%$ for two-stage lithotripsy. The remaining 
Table I. Demographical characteristics.

\begin{tabular}{|c|c|c|c|}
\hline Variable & Senile & Non-senile & P-value \\
\hline Age (years) ${ }^{\mathrm{a}}$ & $69.1 \pm 6.1$ & $41.5 \pm 9.9$ & $<0.01$ \\
\hline \multicolumn{4}{|l|}{$\operatorname{Sex}(n)$} \\
\hline Male & 88 & 185 & \multirow[t]{2}{*}{ NS } \\
\hline Female & 69 & 147 & \\
\hline Body mass index $\left(\mathrm{kg} / \mathrm{m}^{2}\right)^{\mathrm{a}}$ & $23.4 \pm 2.9$ & $22.9 \pm 3.6$ & NS \\
\hline \multicolumn{4}{|l|}{ Intervention history (\%) } \\
\hline ESWL & $32(20.4)$ & $59(17.8)$ & NS \\
\hline PCNL & $17(10.8)$ & $45(13.6)$ & NS \\
\hline fURS & $12(7.6)$ & $31(9.3)$ & NS \\
\hline Preoperative stenting (\%) & $19(12.1)$ & $20(6.0)$ & NS \\
\hline \multicolumn{4}{|l|}{ Comorbidity (\%) } \\
\hline Hypertension & $86(54.8)$ & $74(22.3)$ & $<0.01$ \\
\hline Cardiovascular disease & $26(16.6)$ & $35(10.5)$ & $<0.05$ \\
\hline Anticoagulation & $21(13.4)$ & $23(6.9)$ & $<0.05$ \\
\hline Chronic obstructive pulmonary disease & $27(17.2)$ & $18(5.4)$ & $<0.01$ \\
\hline Morbid obesity & $24(15.3)$ & $73(22.0)$ & NS \\
\hline Diabetes mellitus & $15(9.6)$ & $19(5.7)$ & NS \\
\hline Hydronephrosis & $38(24.2)$ & $70(21.1)$ & NS \\
\hline Urinary tract infection & $21(13.4)$ & $31(9.3)$ & NS \\
\hline Abnormal creatine & $13(8.3)$ & $17(5.1)$ & NS \\
\hline Horseshoe kidney & $2(1.3)$ & $14(4.2)$ & NS \\
\hline Solitary kidney & $2(1.3)$ & $3(0.9)$ & NS \\
\hline \multicolumn{4}{|l|}{ Stone side (n) } \\
\hline Unilateral & 149 & 305 & NS \\
\hline Bilateral & 8 & 27 & NS \\
\hline \multicolumn{4}{|l|}{ Stone location (n) } \\
\hline Upper & 43 & 129 & NS \\
\hline Middle & 55 & 114 & NS \\
\hline Lower & 86 & 181 & NS \\
\hline Pelvis & 61 & 147 & NS \\
\hline Number of stones per side $(n)^{a}$ & $1.5 \pm 1.0$ & $1.6 \pm 0.8$ & NS \\
\hline Mean stone burden $(\mathrm{mm})^{\mathrm{a}}$ & $13.2 \pm 4.9$ & $13.7 \pm 5.8$ & NS \\
\hline Mean digitized surface area $\left(\mathrm{mm}^{2}\right)^{\mathrm{a}}$ & $154.6 \pm 57.7$ & $163.5 \pm 69.3$ & NS \\
\hline UAS successful insertion rate $(\%)$ & $138(87.9)$ & $296(89.2)$ & NS \\
\hline Operative time $(\min )^{\mathrm{a}}$ & $54.6 \pm 13.2$ & $67.1 \pm 16.5$ & NS \\
\hline Single session SFR (\%) & $128(81.5)$ & $246(74.1)$ & NS \\
\hline Overall SFR (\%) & $140(89.2)$ & $284(85.5)$ & NS \\
\hline Hospitalization stay (days) ${ }^{\mathrm{a}}$ & $1.2 \pm 1.6$ & $1.4 \pm 1.2$ & NS \\
\hline \multicolumn{3}{|l|}{ Complication rate $(\%)$} & NS \\
\hline Persistent hematuria & $3(1.9)$ & $5(1.5)$ & NS \\
\hline Ureteral perfortion & $0(0)$ & $0(0)$ & NS \\
\hline Fever & $24(15.3)$ & $43(13.0)$ & NS \\
\hline
\end{tabular}

${ }^{a}$ Data presented as the mean \pm standard deviation. NS, not significant; ESWL, extracorporeal shock wave lithotripsy; PCNL, percutaneous nephroscope lithotripsy; fURS, flexible ureterorenoscopy; UAS, ureteral access sheath; SFR, stone-free rate.

17 patients had postoperative residual stones (diameter ranged from 4 to $5 \mathrm{~mm}$, lower calyceal calculi) and did not take further ESWL treatment. The postoperative follow-up period was 3-24 months. All symptoms of back pain and abdominal pain were eliminated or greatly relieved and hydronephrosis was improved markedly according to KUB or CT. The operation data of the senile group was compared to that of the non-senile group. Operative time and SFR were similar between two 
Table II. Comparison between febrile and afebrile senile patients.

\begin{tabular}{lccc}
\hline Characteristic & Febrile senile & Afebrile senile & P-value \\
\hline Age (years) & $68.5 \pm 5.8$ & $69.2 \pm 6.0$ & $\mathrm{NS}$ \\
Body mass index $\left(\mathrm{kg} / \mathrm{m}^{2}\right)$ & $23.7 \pm 3.0$ & $23.3 \pm 2.8$ & $\mathrm{NS}$ \\
Preoperative stenting $(\%)$ & $4(16.7)$ & $15(11.3)$ & $\mathrm{NS}$ \\
Diabetes mellitus $(\%)$ & $6(25.0)$ & $9(6.8)$ & $<0.05$ \\
Hydronephrosis $(\%)$ & $10(41.7)$ & $28(21.1)$ & $<0.05$ \\
Urinary tract infection $(\%)$ & $8(33.3)$ & $13(9.8)$ & $<0.01$ \\
Mean stone burden $(\mathrm{mm})$ & $13.9 \pm 5.4$ & $13.1 \pm 4.6$ & $\mathrm{NS}$ \\
Mean digitized surface area $\left(\mathrm{mm}^{2}\right)$ & $170.1 \pm 74.6$ & $151.8 \pm 54.9$ & $\mathrm{NS}$ \\
Operative time $($ min) & $62.6 \pm 17.4$ & $53.2 \pm 11.9$ & 133 \\
Total number $(\mathrm{n})$ & 24 & & $<0.05$
\end{tabular}

NS, not significant.

groups, so was the hospitalization length and complication rates (Table $\mathrm{I}$ ).

Within the senile group, between the patients with and without fever, the diabetes mellitus ratio $(\mathrm{P}<0.05)$, hydronephrosis ratio $(\mathrm{P}<0.05)$ and urinary tract infection ratio $(\mathrm{P}<0.01)$, was significantly higher, and the operative time was also significantly longer $(\mathrm{P}<0.01)$ (Table II).

\section{Discussion}

The world's elderly population is growing at a rapid rate. In 2015, people aged $\geq 65$ years accounted for $8.5 \%$ of the world's population, and this proportion is expected to rise to $17 \%$ by the year 2050 (14). Chronic disease, such as hypertension, diabetes and cardiovascular disease, has high prevalence among the elderly. And they are also weaker than the young in cardiopulmonary function, immunity, restorability and so on. On the other hand, percentage of obese patients in the elderly is also increasing gradually with the rising of the living standards. Now a days, the selection of treatment for renal calculi with which kind of minimally invasive method become a prominent question. ESWL or PCNL used to be the choice for the majority of senile patients with kidney stones. ESWL is a kind of non-invasive treatment exhibiting desirable efficacy and safety. However, its SFR is influenced by many factors, such as the size, the location and the texture of stone, the obesity degree of patient and so on. Also, larger stones are often too large to be shattered and require several times of treatment to reach the target size. The reported clearance rate of ESWL for lower calyceal stone is $25-85 \%$ (15). From our experience, it is often very difficult, if not impossible, for the lower calyceal stone to be cleared by ESWL. And the SFR of ESWL for obese patients is also greatly reduced (16). It is reported that, in comparison with the non-senile group treated by ESWL, there was no significant difference in SFR in senile group (17). However, the incidence of complications was higher in senile group than that in non-senile group. Compared with ESWL, the SFR of PCNL is higher, especially for large stones (18). PCNL is an invasive method which is not suitable for relatively weak senile patient with coagulation disorders or poor heart and lung function. The hospitalization time of PCNL is also longer than that of ESWL and URL $(19,20)$. The difficulty of puncture of PCNL in obese patients increases and effect of operation decreases. Berkan used to score and grade on senile patients with kidney stone before PCNL according to Charlson Comorbidity Index. He found that the incidence of complications rose with the score increasing and suggested to take conservative treatment instead of operation for those asymptomatic kidney stone patients with high scores (21).

The technology of fURS has developed rapidly since it was reported by Bagley in 1987 for the first time. fURS has higher SFR for lower calyceal calculi and stone larger than $1 \mathrm{~cm}$ compared with ESWL (22). fURS rarely cause severe arrhythmia which may be induced by ESWL in senile patients with basic rhythm problems and renal colic caused by gravels clogging the ureter after ESWL in some patients (23). fURS has comparable SFR to PCNL, however it has lots of advantages, such as fewer contraindications with a wider range of application, smaller trauma without puncture, less blood loss without potential demand for blood transfusion, shorter hospital stay with quicker recovery (24). Since it is unnecessary to puncture, there are no special requirements for the patient's body shape in fURS. Its therapeutic effect in obese patient is similar to that in patient with normal BMI (25). In our study, all obese patients, including 19 patients with I degree of obesity $(25 \leq \mathrm{BMI}<30)$ and 5 patients with II degree of obesity (BMI $\geq 30$ ), underwent successful operation and there was no significant difference in operation time with patients with normal BMI. Although some patients had poor cardiopulmonary function, there was no relevant accident or complication due to less influence of spinal anesthesia on circulatory/respiratory system compared with general anesthesia.

PolyScope has the advantages of simple operation, clear visibility and a reliable efficacy $(12,26)$. However, the most obvious weakness of this type of flexible ureteroscope is its capability of being able to flex in just one direction. In an empty working channel, the lens barrel has been demonstrated to bend at an angle of 265; the bending angle is reduced by 10 and $2 \%$ respectively, following the insertion of a $3.0 \mathrm{~F}$ stone basket or a $200 \mu \mathrm{m}$ laser fiber (12). Before the insertion of the modular flexible ureteroscope, it was necessary for the UAS to be positioned near the level of the ureteropelvic junction. This aids to reduce the renal pelvic pressure during surgery, serve as protection for 
the lens barrel and provide a convenient access for the extraction of stone fragments (26). In case of ureteral stenosis, it is necessary to indwell an F6 double-J stent for a second operation. It is also necessary for the end of the flexible ureteroscope to be maintained in a stretched state during the insertion of the $220 \mu \mathrm{m}$ holmium laser optical fiber in order to avoid damaging the work channel of the lens barrel and the kidney. When losing the sense of direction in the intracavity, just take out the ureteroscope for reorientation.

For senile patients with kidney stones, the following tips need to be remembered: i) As the majority of senile patients have commodities of hypertension and/or diabetes, their blood pressure and blood sugar need to be monitored closely with systolic pressure under 140-160 $\mathrm{mmHg}$ and blood sugar between 6-10 mmol/1. ii) Operation time need to be controlled strictly, as the immune system of the senile is relatively weak. The plan of stage operation should be made for those patients with large stone. Avoid long-time lithotripsy might reduce the probability of postoperative fever. It is necessary to do the germiculture and sensitivity test before operation and take postoperative prophylactic antibiotic therapy. iii) As many senile patients take antiplatelet drugs or anticoagulant drugs like aspirin and clopidogrel, they need to stop taking the drug for one week to restore their coagulation function. Timely restoration of the use of antiplatelet or anticoagulant agents after the surgery according to the urine color is also necessary. Usually, there is no problem to restore their use 3 days after operation. The duration should be longer if the urine is still red. In particular, urologists should be cautious with the patients with diabetes for many years. Operations should be gentle and meticulous to avoid ureteral mucosa injury, hemorrhage and postoperative infection.

Our experience and suggestions are as follows: i) In consideration of the relatively weak immunity of senile patients, the use of PolyScope can avoid cross infection as it is disposable. ii) The $8 \mathrm{~F}$ diameter lens barrel means the gap between lens barrel and UAS in the PolyScope is larger than that in the traditional fURS with $8.5 \mathrm{~F}$ diameter lens barrel. The large gap brings many advantages, such as sufficient drainage which can reduce intrapelvic pressure effectively, great vortex effects which can accelerate stone extraction and decreased fraction between the lens barrel and the stone which can reduce the damage of lens barrel. iii) We recommend upgrading the PolyScope by adding a 'handle' just like the one used in SMP (27), consisting of a straight and an oblique bifurcated tube. The straight tube can be contiguous with the access sheath and has a receptacle for a silicone or rubber cap at the proximal end. A longitudinal slit can be designed along the axis of the oblique tube to be used as a pressure vent through which the negative aspiration pressure can be adjusted. The end of the oblique tube can be connected to a continuous negative pressure aspirator through clear flexible tubing with the same or larger lumen. A specimen collection bottle can be added between the handle and the aspirator to facilitate stone fragment collection.

Although the quality of fURS has been greatly improved in recent years, it is still not endurable enough to deal with the numerous patents with urolithiasis, especially in a country with a huge population like China. In a multi-center clinical trial, Knudsen et al found that flexible ureteroscope required fixing after its use in 5.3-18 patients (28-30). In addition, according to statistics from multiple articles, the main reasons for repair were the decreased capacity of active bending, the decreased image quality caused by fiber cuts and the sparking of laser in lens barrel (28-32). It is necessary to protect the flexible ureteroscope during operation. The indwelling UAS provides a convenient access point and protection for the lens barrel, benefits for the extraction of stone fragments, also helps to reduce the renal pelvic pressure during surgery which can reduce the intraoperative absorption of lavage fluid and the incidence of postoperative fever and bacteremia $(32,33)$. It is of great significance to adjust the position and curvature of lens barrel according to the ureteral walk, kidney shape, calculi position and calculi size by carefully reading preoperative CTU/IVU (26). The protection of flexible ureteroscope is particularly important in the treatment of lower calyceal calculi as in such circumstance, the flexible ureteroscope needs to maintain a relatively large angle bending state for a long time. In our study, we all take measures of adjusting patients' position and trying to translocate lower calyceal calculi with the stone basket from the lower to the upper or middle calyx to improve the efficiency of the stone breaking. On the one hand, this method can prevent calculi running to the place that the flexible ureteroscope can't reach; on the other hand, it can extend the life of the flexible ureteroscope and reduce equipment losses and maintenance costs by reducing the time of maximal bending.

The removable modular flexible ureteroscopic management with holmium laser lithotripsy has high SFR in senile patients with kidney stone. Given the many advantages of PolyScope such as extensive application, minimal invasion, no blood preparation or transfusion, short hospital stay and reduced maintenance costs, urologists may take into consideration the removable modular flexible ureteroscopic management with holmium laser lithotripsy as the preferred treatment for senile patients with kidney stone.

\section{Acknowledgements}

Not applicable.

\section{Funding}

This study was funded by the Shanghai Municipal Commission of Health and Family Planning Grant (grant no. 201440314) and Shanghai Shenkang Three Year Action Project (grant no. 16CR2030B) and the Shanghai Sailing Program (grant no. 18YF1415200).

\section{Availability of data and materials}

The datasets used and/or analyzed during the current study are available from the corresponding author on reasonable request.

\section{Authors' contributions}

YH was involved in project supervision. BS, KX, JD and YW collected and analyzed the data. YH performed surgical procedures. KX, BS and YW wrote the manuscript. YW and JD corrected the language of the paper. 


\section{Ethics approval and consent to participate}

The Ethics Committee of Xin Hua Hospital Affiliated to Shanghai Jiao Tong University School of Medicine has approved the study and permitted the waiver of written informed consent from patients. The study protocol is also in accordance with the Declaration of Helsinki.

\section{Consent for publication}

Publication of the data in our study does not compromise anonymity or confidentiality or breach local data protection laws.

\section{Competing interests}

The authors declare no potential conflicts of interest.

\section{References}

1. Sorokin I, Mamoulakis C, Miyazawa K, Rodgers A, Talati J and Lotan Y: Epidemiology of stone disease across the world. World J Urol 35: 1301-1320, 2017.

2. Indridason OS, Birgisson S, Edvardsson VO, Sigvaldason H, Sigfusson N and Palsson R: Epidemiology of kidney stones in Iceland: A population-based study. Scand J Urol Nephrol 40 215-220, 2006.

3. Scales CD Jr, Smith AC, Hanley JM and Saigal CS; Urologic Diseases in America Project: Urologic diseases in america, prevalence of kidney stones in the united states. Eur Urol 62: 160-165, 2012.

4. Joo KB, Bae SH and Park YS: Causality of burnout in families with senile dementia elderly using LISREL. Int J Consul Psychol Patients 1: 1-8, 2017

5. Akman T, Binbay M, Sari E, Yuruk E, Tepeler A, Akcay M, Muslumanoglu AY and Tefekli A: Factors affecting bleeding during percutaneous nephrolithotomy: Single surgeon experience. J Endourol 25: 327-333, 2011.

6. Eremenko M, Pink C, Biffar R, Schmidt CO, Ittermann T, Kocher T and Meisel P: Cross-sectional association between physical strength, obesity, periodontitis and number of teeth in a general population. J Clin Periodontol 43: 401-407, 2016.

7. Astrand PO: Human physical fitness with special reference to sex and age. Physiol Rev 36: 307-335, 1956.

8. Michel MS, Trojan L and Rassweiler JJ: Complications in percutaneous nephrolithotomy. Eur Urol 51: 899-906, 2007.

9. Hussain M, Acher P, Penev B and Cynk M: Redefining the limits of flexible ureterorenoscopy. J Endourol 25: 45-49, 2011.

10. Yang B, Ning H, Liu Z, Zhang Y, Yu C, Zhang X, Pan D and Ding K: Safety and efficacy of flexible ureteroscopy in combination with holmium laser lithotripsy for the treatment of bilateral upper urinary tract calculi. Urol Int 98: 418-424, 2017.

11. Cocuzza M, Colombo JR Jr, Cocuzza AL, Mascarenhas F, Vicentini F, Mazzucchi E and Srougi M: Outcomes of flexible ureteroscopic lithotripsy with holmium laser for upper urinary tract calculi. Int Braz J Urol 34: 143-150, 2008.

12. Bader MJ, Gratzke C, Walther S, Schlenker B, Tilki D, Hocaoglu Y, Sroka R, Stief CG and Reich O: The PolyScope: A modular design, semidisposable flexible ureterorenoscope system. J Endourol 24: 1061-1066, 2010.

13. Ding J, Xu D, Cao Q, Huang T, Zhu Y, Huang K, Chen Y, Liang C, Qi J and Huang Y: Comparing the efficacy of a multimodular flexible ureteroscope with its conventional counterpart in the management of renal stones. Urology 86: 224-229, 2015.

14. He W, Goodkind D and Kowal P: An aging world: 2015. Washington, DC: US Government Publishing Office, 2016.

15. Mugiya S: Guidelines on urolithiasis: Update of diagnosis and treatment. Hinyokika Kiyo 58: 703-706, 2012 (In Japanese).

16. Javanmard B, Razaghi MR, Ansari Jafari A and Mazloomfard MM: Flexible ureterorenoscopy versus extracorporeal shock wave lithotripsy for the treatment of renal pelvis stones of 10-20 mm in obese patients. J Lasers Med Sci 6 : $162-166,2015$
17. Chen YZ, Lin WR, Lee CC, Sun FJ, Chow YC, Tsai WK, Chiang PK, Lin TP, Chen M and Chiu AW: Comparison of safety and outcomes of shock wave lithotripsy between elderly and non-elderly patients. Clin Interv Aging 12: 667-672, 2017

18. De S, Autorino R, Kim FJ, Zargar H, Laydner H, Balsamo R, Torricelli FC, Di Palma C, Molina WR, Monga M and De Sio M: Percutaneous nephrolithotomy versus retrograde intrarenal surgery: A systematic review and meta-analysis. Eur Urol 67: 125-137, 2015.

19. Mousavi-Bahar SH, Mehrabi S and Moslemi MK: Percutaneous nephrolithotomy complications in 671 consecutive patients: A single-center experience. Urol J 8: 271-276, 2011.

20. de la Rosette JJ, Opondo D, Daels FP, Giusti G, Serrano A, Kandasami SV, Wolf JS Jr, Grabe M and Gravas S; CROES PCNL Study Group: Categorisation of complications and validation of the Clavien score for percutaneous nephrolithotomy. Eur Urol 62: 246-255, 2012.

21. Resorlu B, Diri A, Atmaca AF, Tuygun C, Oztuna D, Bozkurt OF and Unsal A: Can we avoid percutaneous nephrolithotomy in high-risk elderly patients using the Charlson comorbidity index? Urology 79: 1042-1047, 2012.

22. El-Nahas AR, Ibrahim HM, Youssef RF and Sheir KZ: Flexible ureterorenoscopy versus extracorporeal shock wave lithotripsy for treatment of lower pole stones of 10-20 mm. BJU Int 110: 898-902, 2012.

23. Fankhauser C, Hermanns T, Lieger L, Diethelm O, Müntener M, Umbehr M, Luginbühl T, Sulser T and Poyet C: Comparison of success and complication rates between extracorporeal shock wave lithotripsy (ESWL) and flexible ureterorenoscopy (URS) for untreated renal calculi. Eur Urol Suppl 16: e732, 2017.

24. Zhang $\mathrm{Y}, \mathrm{Yu} \mathrm{CF}$, Jin $\mathrm{SH}, \mathrm{Zhu} \mathrm{H}$ and $\mathrm{Na}$ YQ: A prospective comparative study between minimally invasive percutaneous nephrolithotomy in supine position and flexible ureteroscopy in the management of single large stone in the proximal ureter. Urology 83: 999-1002, 2014.

25. Chew BH, Zavaglia B, Paterson RF, Teichman JM, Lange D, ZappavignaC,MatlagaBR,Nunez-NaterasR,Bruhn A,AltamarHO, et al: A multicenter comparison of the safety and effectiveness of ureteroscopic laser lithotripsy in obese and normal weight patients. J Endourol 27: 710-714, 2013.

26. Gu SP, Huang YT, You ZY, Zhou X, Lu YJ, He CH and Qi J: Clinical effectiveness of the PolyScope ${ }^{\mathrm{TM}}$ endoscope system combined with holmium laser lithotripsy in the treatment of upper urinary calculi with a diameter of less than $2 \mathrm{~cm}$. Exp Ther Med 6: 591-595, 2013.

27. Zeng GH, Wan SP, Zhao ZJ, Zhu J, Tuerxun A, Song C, Zhong L, Liu M, Xu K, Li H, et al: Super-mini percutaneous nephrolithotomy (SMP): A new concept in technique and instrumentation. BJU Int 117: 655-661, 2016.

28. Kramolowsky E, McDowell Z, Moore B, Booth B and Wood N: Cost analysis of flexible ureteroscope repairs: Evaluation of 655 procedures in a community-based practice. J Endourol 30: 254-256, 2016

29. Chi T, Usawachintachit M, Chu C, Allen I, Xu A, Duty B, Sur R, Zaid U, Ramaswamy K, Sorensen M, et al: 1029 Durability of flexible ureteroscopy and predictors of repair: A prospective multi-center study. Eur Urol Suppl 15: e1029, 2016.

30. Knudsen B, Miyaoka R, Shah K, Holden T, Turk TM, Pedro RN, Kriedberg C, Hinck B, Ortiz-Alvarado O and Monga M: Durability of the next-generation flexible fiberoptic ureteroscopes: A randomized prospective multi-institutional clinical trial. Urology 75: 534-538, 2010.

31. Sooriakumaran P, Kaba R, Andrews HO and Buchholz NP: Evaluation of the mechanisms of damage to flexible ureteroscopes and suggestions for ureteroscope preservation. Asian J Androl 7: 433-438, 2005.

32. Traxer O, Dubosq F, Jamali K, Gattegno B and Thibault P: New-generation flexible ureterorenoscopes are more durable than previous ones. Urology 68: 276-281, 2006.

33. Kaplan AG, Lipkin ME, Scales CD Jr and Preminger GM: Use of ureteral access sheaths in ureteroscopy. Nat Rev Urol 13: 135-140, 2016.

This work is licensed under a Creative Commons Attribution-NonCommercial-NoDerivatives 4.0 International (CC BY-NC-ND 4.0) License. 